\title{
Premessa e ringraziamenti
}

Questo libro è il frutto della rielaborazione della mia tesi di dottorato, discussa nell'A.A. 2015/6 presso la "Sapienza Università di Roma". L'idea di occuparmi di un aspetto della lingua di Licofrone mi fu suggerita dal mio maestro Emanuele Dettori e fu bene accolta da Gianfranco Agosti. Essi furono dunque i miei tutores, e non in senso strettamente accademico: da costoro, infatti, ripeto il meglio della mia formazione filologica; né spero di saper pagare il debito di gratitudine che ho contratto con loro.

Durante la preparazione della tesi, ebbi modo di trascorrere un periodo di studio presso il Seminar für Klassische Philologie dell'Università di Heidelberg: qui fui accolto nel migliore dei modi da Athanassios Vergados, con me sempre prodigo di consigli e cortesie.

Sono grato a Enrico Magnelli e Giulio Massimilla per le preziose osservazioni e correzioni con cui hanno arricchito il mio lavoro, oltre che per le parole benevole con cui hanno incoraggiato i miei studi.

Ringrazio infine Marcus Deufert, Heinz-Günther Nesselrath e Peter Scholz per aver accolto il mio lavoro nella collana da loro diretta. Il Professor Nesselrath, in particolare, ha mostrato una volta di più la sua acribia - e la sua pazienza - leggendo e correggendo il mio manoscritto.

C'è appena bisogno di dire che sviste, imprecisioni ed errori sono imputabili a me solo. 
